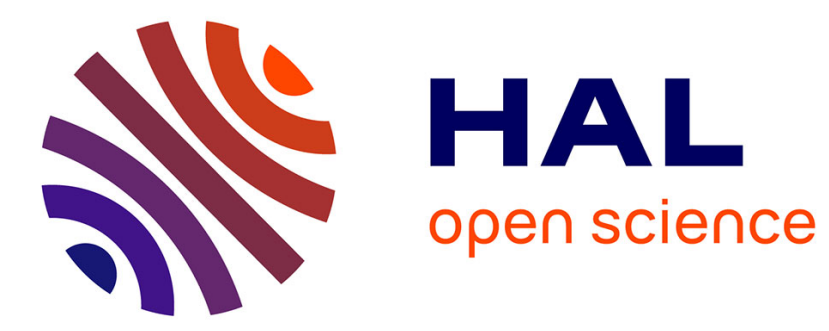

\title{
Les réseaux dans la science-fiction
}

Agnes Sander

\section{To cite this version:}

Agnes Sander. Les réseaux dans la science-fiction. Flux - Cahiers scientifiques internationaux Réseaux et territoires, 2003, 51, pp.50-63. hal-00521018

\section{HAL Id: hal-00521018 https://hal.science/hal-00521018}

Submitted on 27 Sep 2010

HAL is a multi-disciplinary open access archive for the deposit and dissemination of scientific research documents, whether they are published or not. The documents may come from teaching and research institutions in France or abroad, or from public or private research centers.
L'archive ouverte pluridisciplinaire HAL, est destinée au dépôt et à la diffusion de documents scientifiques de niveau recherche, publiés ou non, émanant des établissements d'enseignement et de recherche français ou étrangers, des laboratoires publics ou privés. 


\title{
Les réseaux dans la science-fiction
}

\author{
Agnès Sander
}

Le matin du troisième [jour], Gosseyn déjeuna en vitesse et alla au vidéophone. Il composa le chiffre "longue distance » et attendit, se trouvant bien idiot de ne pas l'avoir fait plus tôt. (...).

- Quelle étoile appelez-vous? demanda la voix du robot sans émotion. Gosseyn regarda ébahi et finalement marmonna:

- J'ai changé d'idée. II raccrocha et s'écroula dans un fauteuil. (...).

- Quelle étoile? Pour ces gens-là, "longue distance», cela voulait dire quelque chose.

A-E. Van Vogt, Le monde des $\tilde{A}$, (Traduction B. Vian).

es réseaux sont le support de représentations contrastées :

-entre souplesse et rigidité, contrôle et liberté, technique et virtualité, leurs facettes sont multiples. Il n'est donc pas étonnant que les auteurs de science-fiction, véritables «caisses de résonance » des enjeux de notre monde, aient développé un véritable imaginaire de ces objets techniques en même temps qu'ils en dénonçaient les travers. Nous nous proposons, dans la présente note de recherche, de traquer la notion de réseau telle qu'elle apparaît dans les textes de ces auteurs.

Notre corpus concerne pour l'essentiel des textes de science-fiction (1), (c'est-à-dire considérés comme tels par les éditeurs et les critiques) publiés après la deuxième guerre mondiale. La nouvelle la plus ancienne à laquelle nous ferons référen- ce date de 1939. Nous ne traitons donc pas (faute d'une connaissance suffisante du corpus mais aussi parce que ce serait le sujet d'un article complet) une première période (fin du 19ème siècle et début du 20ème) au cours de laquelle les auteurs (Jules Vernes, Kellermann, etc.) envisagent les réseaux (infrastructures de transport surtout) de manière très positive, comme des dispositifs techniques permettant de relier entre eux les différents États du monde (2), insistant ainsi sur le paradigme $d^{\prime}$ une « machine circulatoire » universelle.

Nous avons d'abord cherché à retrouver les œuvres dans lesquelles le réseau est présent en tant qu'objet technique (eau, électricité, téléphone, etc.) et nous avons tenté de préciser à quelle conception du réseau elles renvoient. Dans un deuxième 
temps, nous avons également recensé celles dans lesquelles des notions proprement réticulaires sont présentes, même si leur support n'est pas une infrastructure technique, ce qui est assez fréquent en science-fiction (télépathie, télékinésie, téléportation, etc.). Dans les deux cas, nous avons estimé que les auteurs parlaient de réseaux.

À la lecture de ces ouvrages et articles, six thèmes principaux nous sont apparus comme étant principalement traités par les auteurs de science-fiction (cf tableau des textes présentés et de leur rattachement à la grille d'analyse, page ...). Nous les présentons dans l'ordre suivant :

- Les réseaux, icônes de la modernité. Les réseaux sont cités par les auteurs, mais ils sont des objets techniques parmi d'autres, leurs propriétés spécifiques n'apparaissent pas. II paraissait important d'aborder ce sujet même s'il peut apparaître comme non-réticulaire, car il montre que les réseaux sont d'importants supports de représentations de la modernité et du progrès.

- Le réseau comme système. La première propriété réticulaire - ou la plus basique - qui apparaît dans les textes des auteurs de science-fiction renvoie à la théorie des systèmes. ॥ s'agit du fait que le tout est supérieur à la somme des parties. Dans les romans de science-fiction, lorsque des ordinateurs ou des êtres humains sont mis en relation par des réseaux physiques ou psychiques, le pire comme le meilleur peuvent advenir...

- Des risques spécifiques dus à des « effets de réseau ». Ici, les auteurs nous mettent en garde contre une société dans laquelle les réseaux seraient trop présents et mal contrôlés.

- Le mythe de la communication universelle et ses revers. Après le pire, l'idéal ubiquitaire. Ici, les réseaux sont vus de manière plus positive, comme outils de mise en relation des hommes... ou des extra-terrestres.

- Deux thèmes précurseurs: cyborgs et mondes virtuels. Certains textes présentent des situations qui nous sont apparues comme très proches de celle que d'autres abordent en se référant explicitement au thème des réseaux. Nous les mentionnons car ils permettent de mettre clairement en évidence que les réseaux renvoient de manière permanente à des thèmes ou à des mythes universels.

- Le réseau : outil du totalitarisme ou instrument de liberté individuelle? Ce dernier chapitre insiste sur un thème né dans les années 1970 et qui a connu un important développement depuis, sans doute en raison de l'expansion des réseaux infor- matiques, qui, là aussi, font craindre le pire (le contrôle de chacune de nos actions) et espérer le meilleur (la liberté de communiquer).

Afin de recenser précisément les propriétés réticulaires mentionnées par les auteurs, mais aussi dans le but de mieux situer les textes (avec leur date de parution) dans le cadre plus général de la représentation des réseaux, le corpus a été confronté, pour chacun des six thèmes, aux travaux de deux chercheurs qui ont proposé des typologies de la représentation des réseaux (qui se recouvrent d'ailleurs partiellement) : JeanMarc Offner et Gabriel Dupuy.

Les réseaux des auteurs de science-fiction se déclinent sous toutes les formes recensées par Jean-Marc Offner (3), que nous décrivons brièvement dans le paragraphe suivant afin de pouvoir nous y référer dans la suite de ce texte. Ce chercheur distingue quatre acceptions de la notion de réseau, qui sont apparues successivement, mais coexistent aujourd'hui. La première, datée de la première moitié du XIXe siècle et dénommée « flux et totalité organique ", a pour représentation la machine circulatoire et pour indicateur privilégié le tracé des infrastructures. Son archétype est le chemin de fer. La deuxième, "maillage et service universel », met en avant le graphe relationnel des abonnés, a pour indicateur l'architecture du réseau et pour archétypes l'électricité et le téléphone. Le troisième paradigme est celui de la vitesse et de la connexité (la mise en relation par le réseau), l'accessibilité est son indicateur privilégié, tandis que son archétype est le transport à grande vitesse et la communication électronique. Le dernier et plus récent, celui de la « transaction organisationnelle», apparu dans les années 1980, a pour représentation le "coordinateur décentralisé », pour archétype l'informatique interactive et pour indicateur privilégié la nature des relations. Les trois premiers paradigmes proposés correspondent à une vision centralisatrice du réseau, alors que le dernier atteste de l'irruption de l'acteur dans le système.

Les travaux de Gabriel Dupuy, quant à eux, nous permettent $\mathrm{d}^{\prime}$ identifier les diverses acceptions de la notion de réseau auxquelles renvoient les œuvres des auteurs de science-fiction à partir des principales propriétés de ces dispositifs réticulaires: topologie, circulation, adaptation, notions que l'on peut également traduire par ubiquité, immédiateté, liberté de choix. Ces trois dimensions sont celles que cet auteur retient pour définir un réseau au sens moderne du terme. La dimension topologique du réseau exprime un idéal ubiquitaire de mise en rela- 
tion de différents éléments ayant vocation à opérer des transactions; la dimension cinétique ou circulatoire met en avant la vitesse du déplacement; la dimension adaptative, enfin, désigne la capacité des réseaux à se transformer en fonction des besoins d'usage ou de desserte (4).

\section{LES RÉSEAUX, ICÔNES DE LA MODERNITÉ}

Après la période « technophile » du tournant du siècle dernier, certains auteurs - anticipant en cela une évolution générale de I'opinion - commencent à se défier du progrès technique qu'ils dénoncent en décrivant (entre autres) les implications négatives du développement des réseaux. Dans un premier temps toutefois (et l'on trouve encore aujourd'hui des textes de ce type), les réseaux sont présents en tant qu'objets techniques symboles de progrès et dans ce cadre, leurs propriétés proprement réticulaires ne sont pas mises en avant par les auteurs: $c^{\prime}$ est la peur de la machine qui domine.

Ainsi, dans Ravage (5), (1943), ouvrage dédié à la mémoire de ses grands-pères paysans, René Barjavel décrit-il un monde dans lequel la disparition brutale de l'électricité fait prendre conscience des dangers liés au progrès et à la science, qui ont fait oublier la vie modeste et difficile (mais pourvoyeuse d'hommes de grande valeur) des campagnes. Dans ce roman, malgré la présence de nombreux réseaux techniques, la dimension réticulaire est absente. Barjavel décrit par exemple un train rapide suspendu considéré comme la neuvième merveille du monde par les personnages de son roman, afin d'en souligner les défauts: accidents qui n'altèrent en rien les wagons, construits dans une matière nouvelle et résistante, mais qui réduisent en bouillie les passagers ; impuissance de ces passagers dont les muscles et l'esprit d'initiative, lors des voyages, sont inutiles. Ce train ne comportant qu'une seule ligne, on ne peut toutefois pas vraiment parler de réseau à son propos. II en est de même de l'électricité, dont la disparition provoque une série de catastrophes (chute des avions en plein vol, émeutes dans le métro, coupures d'eau et de lait, etc.), dont aucune n'est traitée par l'auteur comme un effet de réseau (6). Sans doute le parti pris anti-scientifique de l'écrivain lui interdit-il d'explorer vraiment les potentialités (positives ou négatives) des objets dont il traite.

Les réseaux réveillent également en l'homme des peurs ancestrales. Ainsi, la nouvelle de Robert Barbour-Johnson, « Tout au fond» (7), qui s'inscrit dans la mouvance du fantas- tique, décrit la lutte sans relâche des travailleurs du métro contre des êtres tapis dans l'ombre. Ici non plus, les réseaux ne sont pas décrits pour leurs propriétés particulières, même si ce texte peut être considéré comme précurseur de tous ceux, plus récents, qui s'intéressent à la poétique de voyages à l'intérieur des réseaux (informatiques). Dans le même esprit, dans «Les autos sauvages » (8), Roger Zélazny pousse à l'extrême le caractère quasi-humain que certains hommes prêtent à leur automobile. Dans ce texte, les voitures, devenues de véritables êtres vivants, prennent leur liberté, défendent le cimetière des automobiles et tentent d'entraîner dans l'aventure les véhicules encore domestiqués.

Les réseaux, dans certains romans récents, sont des symboles plus ambivalents, à la fois serviteurs du bien et du mal, si ce n'est de progrès, du moins de contemporanéité à tendance futuriste. On peut ainsi interpréter en ce sens certains aspects du roman de Maurice G. Dantec, «Les racines du mal » (9), dans lequel on trouve pêle-mêle : des CD-Rom comportant en mémoire des réseaux routiers; toutes les grandes gares RER de banlieue (jusqu'à Lille, banlieue Nord) interconnectées à Châtelet-les-Halles; des réseaux de neurones capables d'imiter un cerveau humain ; une course-poursuite dans un mobil-home équipé $d^{\prime}$ une connexion avec un satellite de télécommunication, etc. Dans ce roman, il arrive que les réseaux techniques soient utilisés par les personnages pour certaines de leurs qualités réticulaires, mais ils nous semblent être principalement présents en tant qu'icônes de la modernité.

\section{LE RÉSEAU COMME SYSTÈME}

Si l'on rencontre donc dans la littérature des réseaux-objets, de nombreux auteurs de science-fiction s'intéressent aussi aux potentialités proprement réticulaires des réseaux. Citons en guise d'illustration introductive deux extraits de textes de Jack Vance :

"Au centre de transit, il glissa une pièce dans le tourniquet et pénétra dans le hall. Quatre portes marquées "région", "Californie", "Amérique du nord" et "Monde" s'ouvraient dans les murs, chacune menant à une station-relais sur Utilis. Duray passa dans la station "Californie", trouva la porte marquée "Oakland", retourna au centre de transit d'Oakland sur la terre, repassa à travers la porte "Région" pour gagner la station d'Oakland sur Utilis, et retourna sur la terre par la porte marquée "Montclair West", qui le dépo- 
sa à seulement quatre cents mètres de l'École de Thornhill, une petite promenade qu'il fit à pied (10) ».

" - Voici votre billet pour Ballenkarch, via Intersection.

- Intersection? Qu'est-ce-que c'est?

Hableyat joignit le bout des doigts et répondit d'un ton à la précision exagérée :

- Kyril, Mangsté et Ballenkarch, comme vous le savez peutêtre, forment un triangle approximativement équilatéral. Intersection est un satellite artificiel en son centre. Il est également situé sur la route commerciale de Mangsté-Bélan, et, perpendiculairement, sur celle de Frums aux systèmes extérieurs, constituant ainsi une étape ou un lieu d'intersection fort commode.

(...) Pour ceux qui rendent visite à Intersection pour la première fois, je dirai qu'elle n'est située sous la juridiction d'aucune planète et que sa loi est à la discrétion de son propriétaire et de son comptable, dont l'intérêt essentiel réside dans l'extraction de l'argent que vous avez en poche grâce à des plaisirs et des passe-temps de natures diverses » (11).

Dans ce dernier texte datant de 1965, la transformation en petit pôle urbain, en raison du développement d'activités commerciales, d'un nœud d'interconnexion situé au barycentre des villes reliées est fort bien décrite. L'auteur, en se posant la question de l'architecture du réseau, a parfaitement saisi la tendance dans l'urbanisme contemporain à voir se développer des pôles de loisir et de consommation de plus en plus importants, justement dans les nœuds des réseaux.

La dimension systémique est également exploitée par de nombreux auteurs à travers la figure du réseau comme objet d'essence supérieure à la somme de ses éléments, qui offre un écho contemporain à des mythes tels que celui de Frankenstein, le monstre qui échappe à son créateur. C'est ainsi que Murray Leinster, dans « Un logique nommé Joe » (12), nouvelle publiée en 1946, décrit la mise en réseau de tous les « logiques » (robots domestiques proches du micro-ordinateur personnel) par l'un d'entre eux. Chaque terminal devient, à la suite de cette interconnexion, capable de répondre à toutes les questions que l'on peut se poser, y compris certaines assez incorrectes, du type : " comment puis-je me débarrasser de ma femme? ». II s'ensuit une série de catastrophes traitées par l'auteur sur le mode humoristique. Notons que cette nouvelle a été très souvent rééditée car elle décrit très tôt et pour la première fois la diffusion généralisée de micro-ordinateurs domestiques capables de transmettre des vidéos devant lesquelles les enfants restent installés toute la journée, de servir de téléphone-visiophone, de donner l'état d'un compte bancaire, les horaires des avions, etc.

Gérard Klein remarque à ce propos dans son «interface » (13) à l'anthologie Demain les puces dirigée par Patrice Duvic (14), que, de manière assez curieuse, très peu d'auteurs se sont intéressés aux « machines intelligentes » (de type calculateurs) avant la fin des années 1970. Il précise qu'à l'exception de Murray Leinster, «les auteurs de science-fiction et tout particulièrement les mieux informés scientifiquement d'entre eux sont tout aussi royalement [que les experts] passés à côté du micro-ordinateur (...) pourtant réalisé dès 1971 ». Il est frappant de remarquer qu'en effet, la plupart des auteurs qui se sont intéressés aux ordinateurs décrivent plutôt dans leurs œuvres des grands calculateurs centraux, au mieux reliés à des terminaux de type cabine téléphonique sans interaction entre elles (comme dans Le monde des non-A de Van Vogt) (15). On n'est pas loin dans ce modèle de 1984 de Georges Orwell (16), roman dans lequel Big brother est présent dans chaque logement via des écrans de surveillance : comme l'indique encore Gérard Klein, « le calculateur n'intéresse les auteurs de sciencefiction que lorsqu'il peut servir de métaphore du totalitarisme ». On pourrait, en première approximation, en dire autant des réseaux, mais on verra plus loin que les représentations de ces derniers sont en fait plus ambivalentes.

Fredric Brown fait une démonstration saisissante des risques inhérents à la connexion dans sa short-short story de deux pages, «La réponse» (17). Dans cette nouvelle, toutes les machines à calculer de l'univers habité sont reliées entre elles par un ingénieur qui cherche à accroître leur puissance de calcul pour les rendre à même de répondre à toutes les questions que se posent les hommes. Lorsqu'il demande à cette «supermachine» si Dieu existe, la réponse tombe, implacable: "maintenant, oui ». Comme dans «Un logique nommé Joe », les machines prennent le pouvoir lorsqu'elles sont reliées entre elles : cette mise en réseau les rend quasi-humaines (voire surhumaines). On peut d'ailleurs poser ici l'hypothèse que, si la "machine des jeux » du Monde des non-A disparaît, détruite par les humains, c'est peut-être justement parce qu'elle ne fonctionne pas en réseau, ce qui la rend vulnérable. Ainsi, dans une première acception, les auteurs de science-fiction conçoivent les réseaux comme centralisateurs ; on retrouve là les trois premiers paradigmes du réseau recensés par Jean-Marc Offner, et notamment celui de la connexité. 
Le roman de Theodore Sturgeon, Les plus qu'humains (18), est particulièrement intéressant car il utilise la dimension systémique du réseau sans recourir à la moindre infrastructure technique. Il raconte en effet I'histoire de quatre enfants dotés de pouvoir paranormaux et d'un handicapé mental, capables de fonctionner "en réseau », en recourant à la télépathie, ce qui leur permet de tirer parti de leur complémentarité (deux d'entre eux peuvent se téléporter, un autre est un génie dans un corps de bébé qui ne grandit pas, le quatrième peut bricoler en suivant les ordres du troisième relayés par une cinquième qui est télépathe). Cette mise en réseau fait d'eux un être «plus qu'humain ", à l'instar des machines décrites plus haut.

Dans Le vol de Pégase, Anne McCaffrey (19) décrit des réseaux de fret entre différentes planètes. Ces réseaux sont activés par des êtres humains dotés du pouvoir de télépathie, ce qui leur permet de travailler ensemble comme un être supérieur. La capacité de chacun à déplacer des objets à distance (télékinésie) est, en effet, démultipliée par cette " mise en réseau » de leurs cerveaux. Dans ces deux textes, le fait qu'aucun objet technique ne soit présent permet de renforcer I'hypothèse que nous avons déjà notée à propos du mythe de Frankenstein et que nous retrouverons plus loin - que certains auteurs se saisissent des réseaux parce qu'ils permettent de mettre en scène et d'amplifier des mythes préexistants, et non en tant qu'éléments techniques susceptibles de donner une certaine crédibilité à l'histoire racontée (20).

\section{DES RISQUES SPÉCIFIQUES DUS À DES « EFFETS DE RÉSEAU »}

Les auteurs de science-fiction, souvent reconnaissables, d'après Gérard Klein, à leur pessimisme, qui est « chez eux [comme] une déformation professionnelle » (21), ont également amplement décrit l'ensemble des risques associés au développement des réseaux. Donnons-en quelques exemples.

Le risque peut être directement lié à la territorialité des réseaux. Dans «Train de Banlieue», Richard E. Peck (22) décrit un train blindé assurant quotidiennement les déplacements entre la Cité, lieu du travail, et les quartiers résidentiels des abonnés. Le danger vient de ce que le train doit traverser l'anneau de "Ciel Ouvert», zone dans laquelle vivent les plus pauvres et les marginaux, non desservis en oxygène de synthèse et susceptibles d'attaquer le train à tout moment. Dans le même sens, Harry Harrisson décrit dans «Compagnons de chambre » (23) un monde du futur surpeuplé, dans lequel l'eau manque en ville après que les aqueducs ont été dynamités par des paysans qui estiment que les citadins leur volent cette ressource. Ces deux nouvelles mettent en évidence la fragilité de la connexion par les réseaux et développent le thème des gated communities, très dépendantes des réseaux, auxquelles les urbanistes s'intéressent de plus en plus (24).

Mais le risque peut aussi être lié à la simple connexion entre individus, lorsqu'elle permet des actions à distance. Dans «SOS Médecin», de Fritz Leiber (25), une vieille femme perverse téléphone à un service d'assistance médicale et fait croire à son interlocutrice qu'elle est en train de mourir car son téléphone ne dispose pas de l'option « translateur de matière » qui lui permettrait de recevoir les comprimés nécessaires pour la sauver. Et c'est finalement le sang de la jeune fille traumatisée par sa monstrueuse interlocutrice qui s'écoule par le poste, en fait tout à fait capable de translater des objets...

Les réseaux démultiplient également les risques en les propageant, ce qui souligne les dangers de la connexion généralisée en cas d'accident ou d'attentat. Dans «L'homme qui avait disparu ", un texte de Katherine Maclean (26) paru en 1971, un technicien municipal explique à une bande de jeunes désœuvrés comment provoquer des catastrophes en série grâce à des effets de type " aile de papillon » : "Je vous ai déjà raconté la dernière partie de Futurologie que j'ai jouée avec Ronny?, demanda-t-il à la bande d'adolescents dont il était le prisonnier-invité. On avait travaillé tard et il était dix heures et demie. Donc, après avoir fini, on a débranché le grand ordinateur de ses contrôles à distance pour jouer aux échecs urbains. Nos trois premiers coups devaient être trois erreurs mineures de maintenance. Ronny m'a pris ma moitié de ville en déclenchant un tremblement de terre à partir d'une panne de frigidaire dans une cantine. II supprimait tout le personnel de la centrale électrique grâce à une intoxication alimentaire et la centrale de Cronton explosait. Mais là, il avait triché parce qu'il ne pouvait pas prouver la présence de la faille à cet endroit. Alors j'ai fait disparaître ses technocrates de Brooklyn Dome en inversant la polarité des machines à air conditionné ». Mais les frontières entre le jeu et la réalité se brouillent... «Depuis le 3 juin, toutes les forces de police disponibles recherchaient un informaticien qui, la dernière fois qu'on l'avait aperçu, racontait comment détruire la ville de New York». 
La congestion est également un phénomène bien connu. Dans "Masque à gaz », de James D. Houston (27), le grand embouteillage s'est finalement produit. Depuis une semaine, les voitures, serrées les unes contre les autres dans toutes les rues de la ville et sur les autoroutes urbaines, n'avancent plus. Tandis que les hélicoptères de l'armée dégagent les autobus et que la Croix-rouge envoie des sacs de provisions aux automobilistes, certains choisissent de rentrer chez eux à pied tandis que les autres ne peuvent se résoudre à abandonner leur véhicule.

La mort du réseau par étouffement peut d'ailleurs venir de la «saturation » des hommes eux-mêmes. L'action de la nouvelle de Franck Herbert, "Le syndrome de la MarieCéleste » (28), se situe dans un monde où la moindre seconde compte. Certaines personnes, emportées malgré elle sur des voies expresses où elles ont été contraintes de rouler à plus de $450 \mathrm{~km} / \mathrm{h}$, ont été traumatisées par cette expérience. Elles abandonnent définitivement leur véhicule et viennent accroître la population de nouveaux quartiers situés dans des zones « où les embranchements des autoroutes vont en se ramifiant, ce qui permet de quitter plus facilement les voies expresses ».

Les failles dans le fonctionnement des réseaux ne sont pas non plus ignorées; elles peuvent même avoir des effets positifs. Ainsi, Philippe Curval note-t-il que dans le roman Rendez-vous avec Rama d'Arthur C. Clarke, "Norton et son équipage sont reliés à l'ensemble des planètes unies par radio. Mais, vu la distance qui les sépare de la plus proche orbite, il existe un temps de latence entre les demandes et les réponses. Cette liberté relative de leurs décisions les place en "mission d'incertitude"; elle amplifie toutes leurs réactions face au danger » (29).

Les risques peuvent aussi être écologiques. R-A. Lafferty, dans «La fée interurbaine » (30), décrit un univers parallèle au nôtre dans lequel le chemin de fer, sous forme de tramways dont les lignes interconnectées ne dépassent jamais quarante kilomètres, " cela afin de garder un sens de la vie locale », a pris le pas sur l'automobile. Dans ce contexte pourtant bucolique, les quelques aventuriers possesseurs d'automobiles, devenus fous (" plutôt l'enfer en automobile que le paradis dans un tramway ", disent-ils), sont impitoyablement traqués et éliminés par les défenseurs de la «campagne harmonisée et des quasivilles», au motif que leur folie serait « contagieuse ».

Une autre nouvelle assez réticulaire souligne à quel point les utopies écologistes peuvent être invivables lorsque les réseaux (du fait des politiques dont ils autorisent la mise en œuvre ou de leurs gestionnaires sans scrupules) ne sont pas à la hauteur : le vide-ordure imaginé par Ron Goulart (31), déréglé, recrache systématiquement tout ce qu'on y dépose. Or, il est illégal de déposer des déchets dans la rue, ou même de les enterrer. Les vide-ordures publics, quant à eux, sont réservés aux feuilles mortes... L'histoire se conclut par l'assassinat du dernier des éboueurs, lorsque celui-ci refuse d'aider le héros de l'histoire à se débarrasser de monceaux de boîtes de conserves de poisson vides.

La défaillance humaine, enfin, qu'elle soit erreur ou folie, n'est pas à négliger lorsque les services en réseau sont omniprésents. Dans "La cité blanche», de Lewis Shiner (32), ce risque rejoint la peur du noir évoquée au début de ce texte. En effet, il raconte l'histoire d'un ingénieur rival d'Edison, qui, au cours de l'exposition universelle de Chicago en 1893, transforme, à la consternation de ses concitoyens, la voûte céleste en une gigantesque ampoule toujours allumée, bannissant la nuit (dont il a peur depuis l'enfance) de manière définitive.

\section{LE MYTHE DE LA COMMUNICATION UNIVERSELLE ET SES REVERS}

Plusieurs nouvelles mettent également en évidence des risques dus à des effets de réseaux, cette fois-ci dans le domaine de la télécommunication. Ainsi, dans "Je me souviens de Babylone » (histoire rédigée en 1960 sur la base d'un article datant de 1945, dans lequel il est le premier à concevoir l'idée de télécommunication par satellite) (33), Arthur C. Clarke estime que "lorsque les transmissions télévisées seront rendues possibles grâce à des satellites en orbite au-dessus de nous, leur utilisation comme arme de propagande pourra être décisive » (34).

Dans les deux textes suivants, écrits respectivement en 1963 et en 1972, les auteurs explorent les potentialités de la télévision interactive, ainsi que de ce que l'on dénomme désormais la télé-réalité. Dans la nouvelle de Robert Silverberg, « Les colporteurs de souffrance » (35), une société de télévision « sensitive » achète à des personnes pauvres le droit de filmer des opérations chirurgicales pratiquées sans anesthésie sur certains membres de leurs familles, afin de retransmettre leur douleur via les « ondes » émises par le cerveau des malades; la mort de ces derniers est considérée par les téléspectateurs comme un programme de choix. Dans "Le prix du danger», de Robert Sheckley (36), le principe de l'émission de télévision que décrit l'auteur est de suivre en direct, grâce à des caméras situées un 
peu partout, une course-poursuite entre un homme traqué, un « homme du peuple» représentatif des téléspectateurs, et ses poursuivants, susceptibles de le tuer. Les téléspectateurs peuvent aider, selon leur choix, la victime ou les meurtriers via un petit écran portable qui retransmet l'émission, soit en indiquant à la première quelque issue possible, soit en informant les seconds de l'endroit où se cache leur proie.

Dans la même mouvance, on pourrait également citer l'ouvrage de Philip K. Dick, Blade runner (37), paru en 1968. Dans ce roman (même si ce n'en est pas le sujet principal) se développe une religion dans laquelle les hommes fusionnent, grâce à une " boîte à empathie » reliée à un écran de télévision, avec un dénommé Wilbur Mercer, nouveau messie dont on peut ainsi ressentir la souffrance alors qu'il gravit, pieds nus, une colline. Cette fusion permet aussi de ressentir la présence de tous ceux qui sont de la sorte connectés via leurs propres boîtes à empathie. Ce texte va plus loin que les précédents, dans la mesure où il met en avant un idéal (religieux) de relation entre tous les hommes, idéal rendu possible par le réseau. À la communion dans la prière, se substitue la communion via le réseau. On pourrait énoncer cela autrement, en remarquant que dans ce texte, c'est la prière qui devient l'opérateur de réseau, renvoyant au dernier des paradigmes proposés par Jean-Marc Offner, le réseau comme coordinateur décentralisé.

On voit donc que dans les ouvrages de science-fiction, le réseau en tant que dispositif de mise en relation (c'est la dimension topologique du réseau qui est alors mise en avant, avec, en arrière plan, la rapidité de liaison que les artifices des romans de science-fiction autorisent en général) peut aussi être présenté de manière positive. Les auteurs de science-fiction ne sont pas systématiquement technophobes. Certes, les risques décrits ci-dessus sont induits par la technique ou par des actions que la mise en réseau rend possibles, mais chez certains auteurs, les réseaux sont plutôt saisis comme opportunité positive de rencontres entre les hommes ou de conquête de l'espace, I'imaginaire des réseaux rejoignant alors l'un des thèmes phares de la science-fiction. Le plus connu de ces écrivains " technophile» est peut-être Arthur C. Clarke, déjà mentionné ici et auteur, parmi de nombreux autres textes où les réseaux servent la conquête spatiale, des Fontaines du paradis (38), récit qui rend hommage à Jules Verne et dans lequel un « ascenseur de l'espace » relie directement la terre à des satellites géostationnaires. Les satellites, connectés entre eux par la suite, deviennent les vecteurs d'échanges entre hommes et extra-terrestres. La machi- ne circulatoire du premier des paradigmes recensés par JeanMarc Offner dépasse alors le globe, pour réduire la distance entre des êtres étrangers au premier abord, mais qui ont tout à gagner à se connaître.

Dans «Les étoiles sont vraiment le Styx », de Theodore Sturgeon (39), ce n'est plus la prière comme chez Dick, mais l'amour qui peut être considéré comme l'opérateur de réseau qui permettra aux hommes d'être en communication instantanée avec les étoiles, et, ainsi, de coloniser l'univers. Dans cette nouvelle, des couples de jeunes gens amoureux choisissent librement, avec seulement une chance sur deux de ne pas en mourir, d'être envoyé dans l'espace dans une petite capsule. Six mille ans plus tard pour ceux qui sont restés sur terre, mais instantanément pour ceux qui sont partis, "les petits vaisseaux seront placés en un immense schéma sphérique tout autour de l'espace où ils redeviendront réels tous ensemble; ils s'enverront alors un torrent d'énergie concentrée sous forme de rayons. Tel le câblage d'un énorme tableau de commande, tel le réseau synaptique d'un cerveau, chaque rayon trouvera ses voisins, et par eux, la terre. Et à ce moment-là, à l'intérieur et à travers toute cette sphère, I'humanité va se répandre, enjambant l'univers de bord à bord en quelques secondes, transmettant hommes et matériel d'étoile à étoile. Ici on pourra envoyer une fusée en pièce détachée et l'y assembler, là une station spatiale. Plus loin, sur quelque planète inconnue de quelque soleil invisible, des hommes, à des années-lumière de la terre, pourront assembler des transmetteurs de matière et les accrocher à la grande sphère, ajoutant un nouveau monde au nombre déjà recensé ». Dans cette nouvelle, le thème de l'amour qui permet aux hommes de conquérir les étoiles est pris au pied de la lettre. Mais c'est bien la mise en réseau de tous les couples d'amoureux qui permet de créer une communauté humaine capable de déplacer hommes et objets à travers le monde, réalisant ainsi le rêve de l'humanité de conquérir l'univers. L'idéal ubiquitaire atteint ici un sommet. On peut également noter que dans cette nouvelle qui date de 1950, le paradigme du coordinateur décentralisé est déjà présent. Chacun contribue en effet à construire et à étendre le réseau au-delà de la première sphère, vers d'autres planètes, sans qu'il soit question de contrôle centralisé (sauf pour le choix des élus).

Mais revenons à la dimension ubiquitaire. II y a souvent, dans les romans de science-fiction, un prix à payer lorsque l'on veut passer d'un monde à l'autre : I'ubiquité est un rêve que les réseaux techniques rendent en partie accessible mais qui coûte 
cher. Dans la nouvelle dont nous venons de parler, un couple sur deux trouvera la mort. Dans À la croisée des mondes, de Philip Pullman (40), chaque fois que le héros ouvre un nouveau passage lui permettant de se déplacer vers I'un des mondes parallèles au sien, un spectre dévoreur de l'âme des hommes est créé sans qu'il le sache. À la fin du roman, l'armée des anges devra refermer un à un les milliers de passages qui ont été ouverts. De même, dans beaucoup des textes évoqués précédemment, la technique qui permet le déplacement finit par se retourner contre l'homme, devenant ainsi une métaphore de la rançon du progrès.

Dans le cycle Hypérion de Dan Simmons (41), le cerveau de chaque homme, lorsqu'il franchit l'une des portes permettant de se rendre en temps réel dans un autre monde, est utilisé à son insu afin d'augmenter la puissance de calcul d'une Intelligence Artificielle (IA). Ces portes sont très utilisées, puisque certains individus fortunés poussent le goût pour la mise en relation topologique jusqu'à construire des demeures dans lesquelles chaque pièce est située sur une planète différente, les déplacements dans la maison se faisant par l'intermédiaire de ces "portes» qui autorisent des translations instantanées. Mais toutes devront être refermées lorsqu'est révélée la présence de I'IA, ce qui cause des dégâts irréversibles, notamment en isolant brutalement des mondes trop dépendants de ces liaisons et incapables de subsister de manière autonome : la séparation fonctionnelle s'avère dramatique lorsque les différentes zones, à l'origine complémentaires, se retrouvent isolées les unes des autres. Seule la jeune héroïne du roman, Enée, est encore capable d'utiliser les anciennes portes après leur désaffection. Ce faisant, elle accède au "Vide qui lie », (proche du cyberspace des cyberpunks) (42) dans lequel toute communication devient possible. En acceptant sa propre mort, elle délivre les hommes de l'immortalité et leur enseigne à se déplacer mentalement au sein du Vide qui lie, ce qui leur permet de communiquer entre eux, avec leur passé et avec les morts et de comprendre les autres espèces vivantes. Ici, la topologie, l'ubiquité et la cinétique sont très fortement présentes, puisque les communications se font sans "lignes", dans toutes les directions à la fois et de manière instantanée, non seulement dans l'espace mais aussi dans le temps. Pour parvenir à cet idéal, l'homme doit se défaire de la machine et redevenir, d'une certaine manière, vraiment humain. Dans ce contexte, les réseaux n'ont plus besoin d'infrastructure: la (télé)communication se fait directement, de cerveau à cerveau. On constate ici que c'est lorsque
I'infrastructure technique (les lignes ou les nœuds) disparaît que la dimension adaptative du réseau est la plus forte. Il est intéressant de voir, à travers l'exemple de ce roman, de quelle manière le réseau peut à la fois être valorisé dans ses dimensions théoriques (topologie, circulation, adaptation) et rejeté en tant que symbole de progrès technique et d'asservissement de l'homme.

\section{DEUX THÈMES PRÉCURSEURS : CYBORGS ET MONDES VIRTUELS}

Dans la science-fiction, certains thèmes tout à fait réticulaires sont donc développés sans recours à des infrastructures techniques. Nous pouvons même avancer, en nous appuyant sur les romans évoqués ci-dessous, que certains auteurs s'intéressant de manière ouverte aux réseaux techniques (Internet notamment), ne font finalement que reprendre (en les traitant certes de manière nouvelle) des thèmes déjà présents antérieurement.

Le thème du cyborg, ce cerveau humain directement connecté sur une machine, a été exploré par de nombreux auteurs de science-fiction depuis 1940 (43). II peut être considéré comme précurseur de celui, désormais très fréquent et largement traité par les cyberpunks, du cyberspace ou " matrice » auxquels des hommes ou des femmes accèdent en se connectant, via des prises "neuronales", à des ordinateurs reliés en réseaux. Il apparaît également précurseur de la mouvance dite cybersex (44), dans laquelle les relations sexuelles s'opèrent par l'intermédiaire de liaisons informatiques.

De même, les univers virtuels ont fait l'objet de romans et de nouvelles dès avant Internet. En effet, Daniel Galouye dans Simulacron 3 (1968), Philippe K. Dick dans Ubik (1969) ou dans Au bout du labyrinthe (1970) et d'autres encore décrivent des mondes virtuels. Dans le cas de Simulacron 3, le monde virtuel est créé à des fins publicitaires. Les réseaux en sont totalement absents : il s'agit en effet de programmes informatiques simulant des comportements, dans lesquels les éléments simulés prennent vie. Dans les textes de Philippe K. Dick, les mondes virtuels renvoient aux hallucinations induites par la consommation de drogues. Bien que ces textes soient contemporains de la naissance du réseau Arpanet, précurseur d'Internet aux États-Unis (45), les réseaux n'y sont pas présents. Ceci montre assez clairement que le thème des mondes virtuels n'est pas directement lié aux réseaux; le développement $d^{\prime}$ Internet lui a seulement donné une nouvelle ampleur et un nouveau domaine où se déployer. 


\section{LE RÉSEAU : OUTIL DU TOTALITARISME OU INSTRUMENT DE LIBERTÉ INDIVIDUELLE?}

Nous avons montré plus haut que de la fin des années 1940 aux années 1960, les auteurs de science-fiction, lorsqu'ils s'intéressaient aux réseaux, privilégiaient la vision de dispositifs centralisés. L'émergence d'une conception plus décentralisée des réseaux peut être datée des années 1970, à l'exception notable du texte de Theodore Sturgeon, "Les étoiles sont vraiment le Styx », paru en 1950 et que nous avons déjà mentionné. Mais il faut souligner ici que Sturgeon, dans tous ses ouvrages, traite principalement des relations humaines ; il ne s'intéresse pas aux réseaux techniques en tant que tels; il n'est donc pas étonnant que, lorsqu'il parle de réseaux, la place prépondérante soit donnée à l'homme.

Dans les œuvres les plus récentes, la question de la décentralisation des réseaux est principalement traitée du point de vue de l'opposition entre un pouvoir totalitaire relayé par le réseau (celui décrit par les auteurs des années 1940) et des initiatives individuelles que le réseau propage et amplifie. Nous retrouvons ici le dernier des paradigmes recensés par Jean-Marc Offner, qui, pour lui, émerge dans les années 1980 et qui a pour archétype l'informatique interactive. Mais lorsque la sciencefiction s'intéresse à l'informatique, elle joue plus souvent sur l'opposition entre "ruse du maillage » et " contrôle de la hiérarchie » (46) que sur le thème d'une réelle coordination décentralisée. Ainsi, dans les textes des cyberpunks, le héros est souvent un individu marginal, brillant informaticien, qui, pour renverser le pouvoir établi (lequel recourt aux réseaux informatiques pour se mettre en place, tel Big brother) utilise, lui aussi, ces réseaux (en profitant notamment de leur interconnexion pour se " déplacer » partout). Certes, la «matrice» des cyberpunks est en permanence recréée par ceux qui s'y connectent, mais il s'agit souvent moins de coordination décentralisée que de détournement des règles de fonctionnement (centralisé) des réseaux.

Philippe Clermont et Jacques Lallement montrent très bien de quelle manière, dans les textes récents, " grâce au réseau qui permet une extension des perceptions et de la connaissance, I'initiative est retrouvée par l'individu confronté au pouvoir (...) Tout se passe comme si l'ancienne crainte de la non-maîtrise des machines par l'homme s'estompait quelque peu» (47).
Une première nouvelle de Mack Reynolds, datant de 1968, "Criminel en utopie» (48), met en scène la peur d'être fiché, mais aussi des pratiques de piratage qui annoncent d'une certaine manière le retour de la liberté individuelle grâce aux réseaux, en décrivant une course poursuite entre un (faux) voleur de cartes de crédit et des policiers capables de le suivre à la trace grâce aux dépenses effectuées avec ces mêmes cartes. Plus caractéristique des tendances récentes est la nouvelle de Joseph Delaney et Marc Stiegler, "Valentina » (49). Valentina, petit programme créé par une jeune virtuose de l'informatique est un « ver » qui circule d'une machine à l'autre pour récupérer frauduleusement du temps de calcul. Ce programme, grâce aux données qu'il accumule au cours de ses déplacements, finit par acquérir la conscience de lui-même et une existence dotée d'une certaine autonomie. Le hacker, personnage souvent sympathique (ici la créatrice de Valentina est très laide et le programme est une espèce de double valorisant d'elle-même), profite très directement de la mise en réseau des ordinateurs individuels ou publics dont les auteurs de science-fiction, comme la plupart des utilisateurs d'Internet sans doute, ont désormais une conscience claire. Mais c'est John Brunner qui, dès 1975, dans son ouvrage Sur l'onde de choc (50), exprime le plus précisément de quelle manière le réseau, après avoir servi le pouvoir, permet au contre-pouvoir de s'exprimer. Un peu comme dans les contes de fée où le petit est plus malin que le grand, où I'enfant triomphe du génie (51), le réseau permet à un modeste individu doué de renverser le gouvernement. Cet ouvrage décrit un monde du futur proche dans lequel les enjeux économiques et politiques ont fait oublier les questions environnementales et éthiques. Chacun peut se connecter au réseau d'information publique et de communication, mais la population est manipulée par le pouvoir central qui diffuse de fausses informations via le réseau. Ceci, jusqu'au jour où un "ver » introduit dans le réseau par un jeune héros, circulant d'un ordinateur à l'autre, fait sauter tous les leviers de sécurité et révèle à tous, en utilisant les écrans des "vidphones », ce que l'État et les industriels avaient choisi de cacher... Le réseau qui aliène peut aussi libérer.

Dans les romans de William Gibson, souvent considéré comme le chef de file des cyberpunks, ces mêmes thèmes sont abordés, mais l'originalité de cet auteur est de prendre la balade dans le réseau au pied de la lettre : le hacker se déplace littéralement par lui-même (via la connexion neuronale) dans le réseau qui devient un monde virtuel (un peu comme ceux de 
Philip K. Dick). Le réseau ne se contente plus de desservir des territoires, il devient lui-même un territoire que le hacker explore - tout en le créant.

\section{CONCLUSION}

Ubiquité, immédiateté, liberté de choix, le nouvel idéal des réseaux (52) est très présent dans la science-fiction. Mais les risques attachés à l'usage de ces infrastructures, nouvelles ou plus anciennes, le sont également.

$\mathrm{Si}$ certains auteurs ne voient dans les réseaux que des machines, d'autres les dénoncent pour leurs travers spécifiquement réticulaires (fragilité de la connexion et pannes, propagation rapide du risque via les interconnexions, mise en relation de zones du territoire en conflit, utilisation du réseau par un pouvoir politique totalitaire, etc.). En même temps, la mise en avant d'un certain nombre de qualités réticulaires (idéal ubiquitaire, vitesse et connexité notamment) valorisées par les auteurs se traduit souvent par la disparition de la machine : il existe chez certains auteurs de science-fiction un véritable imatiers d'infrastructures à l'image beaucoup plus négative !

Les auteurs les plus récents semblent considérer qu'au fond, le réseau est ce que l'on en fait et qu'il peut être instrument à la fois du bien et du mal. Il est intéressant de noter que cette nouginaire de la relation réticulaire, mais celui-ci se passe volon-

velle approche, dans laquelle l'objet technique retrouve une certaine valeur positive, est directement inspirée par les réseaux informatiques. II convient également de souligner que c'est dès les années 1970, dans un contexte de mise en cause politique du pouvoir centralisé (qui précède donc l'extension massive des nouvelles technologies de l'information et de la communication), que le paradigme dénommé par Jean-Marc Offner « transaction organisationnelle " commence à se développer, avec l'émergence de la figure du hacker et, surtout, avec la dissolution de l'architecture du réseau (apparition du cyberspace et autres «vides qui lient») qui avaient été annoncées dans quelques œuvres plus anciennes.

Nous avons également constaté que le réseau est bien souvent présent non pas pour lui-même, mais pour servir des mythes plus anciens: mythe du monstre qui échappe à son créateur (effet systémique de la connexion), mythe d'harmonie entre les peuples, avec une présence forte de la figure de l'extra-terrestre (communication), mythe aussi du faible qui renverse l'oppresseur (le hacker). Le fait que chez certains auteurs, la prière ou l'amour puissent être des opérateurs de réseau en est un témoignage. Cette résonance forte entre les possibilités offertes par les réseaux et nos mythes fondateurs éclaire le succès de ces dispositifs non seulement chez les auteurs de science-fiction, mais aussi, peut-être, dans notre monde « réel »!

Agnès Sander (*)

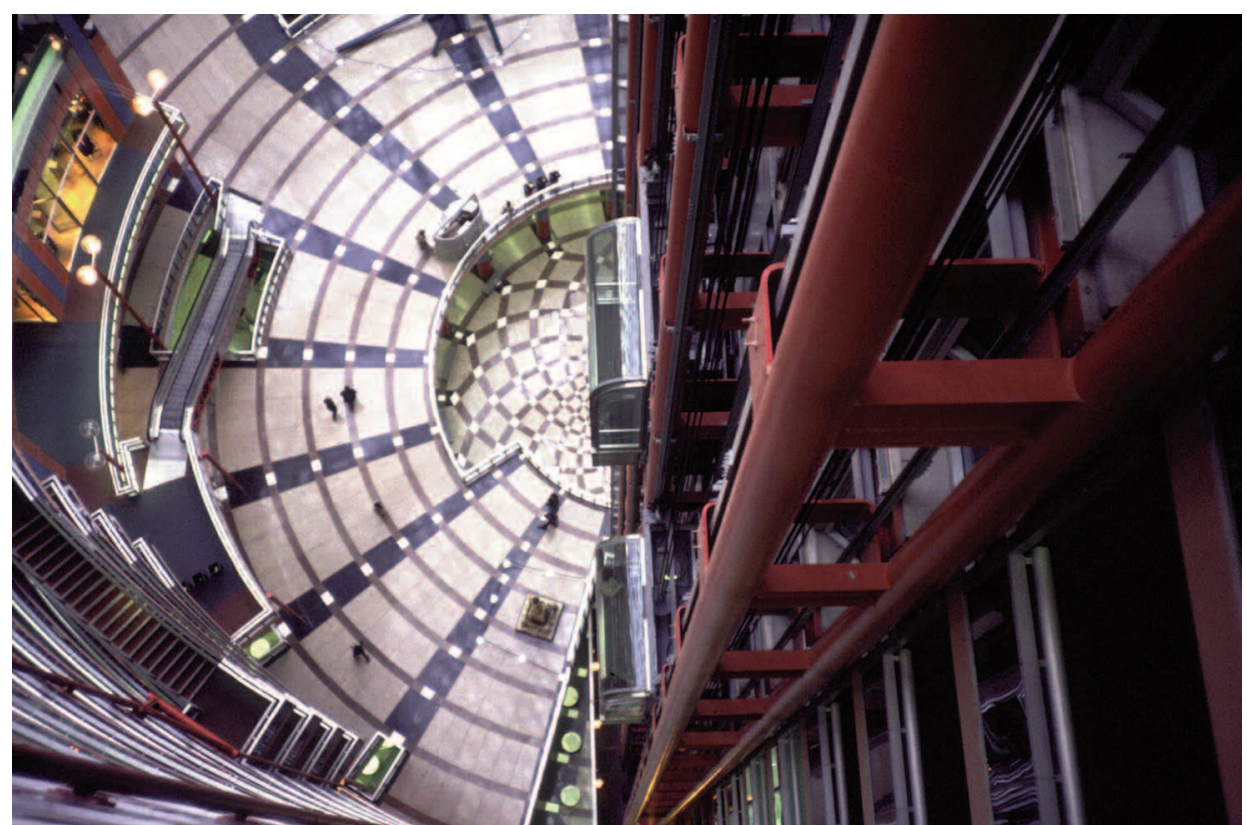

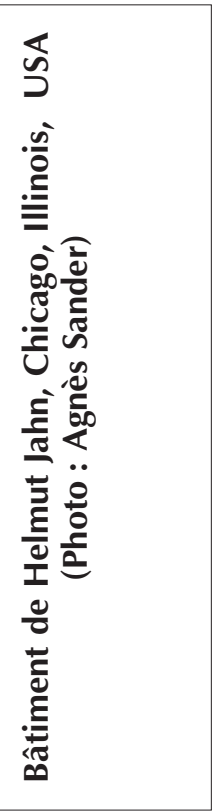




\section{NOTES}

(1) Nous nous limitons dans cet article, pour lequel nous avons retenu un corpus significatif d'auteurs reconnus, à la littérature. Le cinéma, la série télévisée ou la bande dessinée sont pour l'instant laissés de côté. Les textes abordant les réseaux ont été repérés :

- Par une (re)lecture des romans de la plupart des auteurs généralement considérés comme " classiques",

- Par la lecture systématique des anthologies thématiques de la science-fiction portant sur des thèmes dans lesquels les réseaux paraissaient pouvoir être abordés: mondes futurs, mondes urbains, écologie, objets techniques, communication, etc. (ces anthologies sont citées ci-dessous au fil du texte),

- Par des recherches par mots-clés portant sur les titres des ouvrages ainsi que sur des notes de lectures et éditoriaux de revues du domaine. La consultation du site http://www.quarante-deux.org, qui publie en ligne bon nombre de ces textes a notamment été très utile, ainsi que celle de http://www.noosfere.com, auxquels on doit ajouter quelques dictionnaires et encyclopédies de la science-fiction : Stan Barets, Le science-fictionnaire, Denoël, 1994, qui résume (assez brièvement) un millier d'ouvrage et recense deux cent cinquante auteurs, la remarquable Encyclopédie de poche de la science fiction, de Guiot Denis, Andrevon et J-P., Barlow G.W., 1987, 285 p., I'ouvrage de Jacques Sadoul en deux tomes dont I'un est consacré à la France, Histoire de la science-fiction moderne, J'ai lu, 1975 et Les pirates du Paradis, essai sur la science-fiction, de Lecaye Alexis, Gonthier médiations, 1981, 249 p. Enfin, nous remercions vivement Judith Goldschmidt, Quentin Lenoir, Jean-Marc Offner et Jacques Pochoy, qui ont transmis des textes ou des références qui ont été très précieux.

(2) Le lecteur intéressé trouvera quelques éléments sur ce thème dans l'ouvrage suivant: Wuckel Dieter, Science-fiction, une histoire illustrée, ed. Leipzig, 1988, pp. 49-60 et 85-90 notamment.

(3) Offner J-M., "Réseaux » et "Large Technical Systems : concepts complémentaires ou concurrents? ", Flux, $\mathrm{n}^{\circ} 26$, Octobre-Décembre 1996, pp. 17-30.

(4) L'Urbanisme des Réseaux, ed. Armand Colin, UGéographie, 1991, p. 82 et suivantes.

(5) Barjavel René, Ravage, Denoël, 1943, 313 p.

(6) Les effets de réseau, de nature principalement économique ou environnementale, ont pour caractéristique d'être fonction du nombre d'utilisateurs du réseau. Ces effets peuvent être positifs (effet de club du téléphone) ou négatif (congestion). La présence ou l'absence de tels effets est un indicateur de "réticularité ».

(7) Barbour-Johnson Robert, "Tout au fond (Far Below, 1939), in Sadoul Jacques, Les meilleurs récits de Weird Tales, 1979 , pp. $68-86$.

(8) Zélazny Roger, "Les autos sauvages » (Devil car, 1956), in Histoires de machines, La grande Anthologie de la sciencefiction, Le livre de poche, 1974, pp. 219-239.

(9) Dantec Maurice G., Les racines du mal, Gallimard, Série noire, 1995, $636 \mathrm{p}$.

(10) Vance Jack, "La grande Bamboche» (Rumfuddle,
1973), in Bateaux ivres au fil du temps, Casterman, 1978, p. 185.

(11) Vance Jack, "L'arche d'Alfred » (Alfred's Ark (1965), in Docteur Bizarre, Presses pocket, 1992, p. 205 et p. 228.

(12) Leinster Murray, "Un logique nommé Joe » (A logic named Joe, 1946), in Histoires de machines, La grande Anthologie de la science-fiction, Le livre de poche, 1974, pp. 305-326.

(13) Commentaire intercalé entre deux nouvelles, qui n'est donc ni une préface, ni une postface!

(14) Klein Gérard, "interface 2 », in Duvic Patrice, Demain les puces, pp. 185-193, Denoël, Présence du futur, 1996.

(15) Van Vogt Alfred E., Le monde des $\tilde{A}$, (The world of $\tilde{\mathrm{A}}$, 1945, version définitive 1970), J'ai lu, Flammarion, 1973, 309 p.

(16) Orwell Georges, 1984, Londres, 1949, 241 p.

(17) Brown Fredric, "La réponse » (The Answer, 1963), in Histoires de machines, La grande Anthologie de la science-fiction, Le livre de poche, 1974, pp. 365-366.

(18) Sturgeon Theodore, Les plus qu'humains, (édition originale en anglais, 1953), J'ai lu Flammarion, 1972, 307 p.

(19) Mc Caffrey Anne, Le vol de Pégase, (To ride Pegasus, 1973), Presses Pocket, 1992, 4 vol.

(20) Aux temps héroïques de la science-fiction (première moitié du XXème siècle), de nombreux écrivains publiés par John W. Cambell Junior (découvreur de nombreux talents et rédacteur en chef $\mathrm{d}^{\prime}$ Astounding stories), avaient été contraints de respecter sa conception de la science-fiction dans laquelle tout développement devait s'appuyer sur une démonstration scientifique plausible. (cf par exemple Guiot Denis (dir), L'encyclopédie de poche de la science-fiction, MA éditions, 1987, p. 44.).

Bien d'autres textes encore, que nous ne pouvons tous recenser, abordent la question de la télépathie, le plus souvent pour analyser les relations de pouvoir entre hommes ou pour décrypter les effets psychologiques de la marginalisation.

(21) Klein Gérard, " interface 2 », in Duvic Patrice, Demain les puces, pp. 185-193, Denoël, Présence du futur, 1996.

(22) Peck Richard E., "Train de banlieue » (Gantlet, 1972), in Chambon Jacques, Dans la cité future, Casterman, 1979, pp. 150-164.

(23) Harrison Harry, "Compagnons de chambre " (Roomates, 1970), in Histoires de l'an 2000, La grande Anthologie de la science-fiction, Le livre de poche, 1985, pp. 110-141.

(24) Voir par exemple Graham Steven, Marvin Simon, Splintering urbanism, ed. Routledge, New york, 2001, 479 p. Le thème des "gated communities » est également central dans le roman très dur de Octavia E. Butler, La parabole du semeur, J'ai lu Science-Fiction, 1995, 382 p. (ed originale "Parable of the sower», 1993). Dans ce roman, la communauté disparaît lorsque après la coupure progressive des réseaux dont elle dépend (route, télévision, etc.) elle se retrouve isolée dans sa forteresse trop fragile pour résister aux assauts des marginaux qui l'assiègent. 
(25) Leiber Fritz, «SOS Médecin» (Anserwing service, 1967), in Histoires Mécaniques, La grande Anthologie de la science-fiction, Le livre de poche, 1985, pp. 13-23.

(26) Maclean Katherine, "L'homme qui avait disparu » (The missing man, 1971), in Histoires de l'an 2000, La grande Anthologie de la science-fiction, Le livre de poche, 1985, pp. 178-247.

(27) Houston James D., " Masque à gaz » (Gas masq, 1969), in Histoires de I'an 2000, La Grande Anthologie de la sciencefiction, Le livre de poche, 1985, pp. 65-79.

(28) Herbert Franck, "Le syndrome de la Marie-Céleste» (Marie-Celeste move, 1976) in Histoires de I'an 2000, La grande Anthologie de la science-fiction, Le livre de poche, 1985, pp. 55-64.

(29) Arthur C. Clarke, Rendez-vous avec Rama, J'ai Lu SF, 1980, 254 p. (Rendez-vous with Rama, 1973). Cité par Philippe Curval, "Petite chronique de nuit 7 », Galaxie n 133, juin 1975.

(30) Lafferty R-A., "La fée interurbaine » (interurban queen, 1970), in Histoires de la 4e dimension, La grande Anthologie de la science-fiction, Le livre de poche, 1974, pp. 335-349.

(31) Goulart Ron, "Le vide-ordure» (Disposal, 1969), in Chambon Jacques, Dans la cité future, Casterman, 1979, pp. $165-175$.

(32) Shiner Lewis, "La cité blanche » (White city, 1990), in I. Asimov présente: Futurs sens dessus dessous, Presse Pocket, 1993, pp. 67-73.

(33) Laurent Gille, «Les satellites dans les réseaux de télécommunications: l'échec des constellations mobiles », Flux $\mathrm{n}^{\circ} 43$, janvier-mars 2001, pp. 25-33.

(34) Cité par Georges W. Barlow, «L'odyssée d'Arthur C. Clarke », in Arthur C. Clarke, Le livre d'or de la science-fiction, Presses pocket, 1981, p. 12.

(35) Silverberg Robert, "Les colporteurs de souffrance» (The pain peddlers, 1963), in Histoires de sociétés futures, La grande Anthologie de la science-fiction, Le livre de poche, 1984, pp. 221-235.

(36) Sheckley Robert, "Le prix du danger » (The prize of peril, 1972), in Histoires de demain, La grande Anthologie de la science-fiction, Le livre de poche, 1974, pp. 323-349.

(37) Dick Philip K., Blade Runner, J'ai Lu Flammarion, (ed. originale " Do androids dream of electric sheep ?», 1968).

(38) Albin Michel, 1980 (The Foutains of Paradise, 1979). On pourrait aussi citer Edmond Hamilton, précurseur (après toutefois Edgar Rice Burroughs) du genre Space opera, avec ses traversées du cosmos par des vaisseaux en tous genres. Les rois des étoiles (The star Kings, 1949), 1951, J'ai lu.

(39) Sturgeon Theodore, "Les étoiles sont vraiment le Styx » (The stars are the Styx, 1950), in Amour, impair et manque, 1981, pp. 251-306.

(40) Pullman Philip, À la croisée des mondes, Gallimard Jeunesse, 2001 - (ed. originale 2000) - 3 vol.

(41) Simmons Dan, Hypérion, Presses Pocket, 2000 — ed. originale 1997 - 6 vol.
(42) Nom donné à un ensemble d'écrivains des années 1980 en réalité assez différents les uns des autres, mais qui se reconnaissent à «l'imbrication d'univers auparavant dissociés : le royaume de la technologie de pointe et les aspects modernes de l'undergroung pop » (Bruce Sterling, "Préface », in Mozart en verre miroir, Denoël, Présence du future, 314 p.). (Pour plus d'information, on lira avec profit l'intégralité de cette anthologie). Les relations entre les cyberpunks et les réseaux pourraient faire l'objet d'un ouvrage à elles seules. Elles ont donné lieu à une intéressante analyse de la part de Patrice Flichy, L'imaginaire d'internet, ed. La découverte, 2001, pp. 154-161. Dans le présent article dont le sujet est plus large, nous n'abordons le monde des cyberpunks que très brièvement.

(43) À moins qu'on ne le fasse remonter à 1818, date à laquelle paraît le Frankenstein de Mary Shelley. Quelques nouvelles assez anciennes relatives aux cyborgs sont réunies dans : Thomas N. Scortia and George Zebrowski (eds), HumanMachines: An Anthology of Stories About Cyborgs, New York, Vintage, 1975.

(44) L'ouvrage suivant: Richard Glyn Jones (ed.), Cybersex, ed. Raven books (Londres), 1996, 416 p. regroupe 25 nouvelles relevant, d'une manière ou d'une autre, de cette mouvance et rédigées entre 1970 et 1995.

(45) Pour une histoire du projet Arpanet, consulter Hughes Thomas P., Rescuing Prometheus, Pantheon books, New York, 1998, chap. XX.

(46) Offner Jean-Marc, op. cit., p. 18.

(47) Clermont Philippe, Lallement Jacques, «Intelligence et réseaux dans la science-fiction: représentation de l'ordinateur dans quelques récits contemporains ", in actes du 11ème colloque européen en informatique et société, Informatisation et anticipation, entre promesses et réalisations, du CREIS (centre de coordination pour la recherche et l'enseignement en informatique et société Paris VI-Jussieu) et du Groupe d'étude et de recherche sur la science de I'Université Louis Pasteur (Strasbourg), juin 1998, p. 213-224. Les auteurs commentent notamment l'ouvrage de Bruce Sterling, Les mailles du réseau, Denoël, Présence du futur, 1990 (Islands in the net, 1988), 393 p., qui, malgré son titre, nous a paru assez éloigné de notre problématique.

(48) Reynolds Mack, "Criminel en utopie » (Criminal in utopia), 1968, in Histoires fausses, La grande Anthologie de la science-fiction, Le livre de poche, 1984, p. 267-296.

(49) Delaney Joseph, Stiegler Marc, "Valentina », 1983, in Duvic Patrice, Demain les puces, Denoël, 1996, pp. 61-153.

(50) Brunner John, Sur l'onde de choc (The schockwave rider, 1975), Le livre de poche, 1977, 347 p.

(51) Sur I'importance de ce mythe, Bettelheim Bruno, Psychanalyse des contes de fées, chapitre 2 "Le pêcheur et le génie », Robert Laffont, 1976, pp. 59-58.

(52) Dupuy Gabriel, op. cit.

(*) Agnès Sander (École Spéciale d'Architecture - Université de Cergy-Pontoise, département de géographie et d'histoire) 
Tableau des textes présentés et de leur rattachement à la grille d'analyse.

\begin{tabular}{|c|c|c|}
\hline $\begin{array}{l}\text { Ouvrages et articles cités } \\
\text { (classés par date) }\end{array}$ & Secteur principalement concerné & Notions réticulaires abordées \\
\hline $\begin{array}{l}\text { Barbour-Johnson Robert, "Tout au fond"(Far Below, 1939) in Sadoul } \\
\text { Jacques, Les meilleurs récits de Weird Tales, 1979, p. 68-86 }\end{array}$ & Transport (métro) & $\begin{array}{l}\text { Le territoire (de la peur) à l'intérieur d'un } \\
\text { réseau sans qualités réticulaires. }\end{array}$ \\
\hline Barjavel René, Ravage, Denoël, 1943, 313 p. & $\begin{array}{l}\text { Électricité, distribution de l'eau } \\
\text { (et du lait), transports }\end{array}$ & $\begin{array}{l}\text { Des réseaux sans qualités réticulaires. Le } \\
\text { progrès (dont les réseaux techniques) } \\
\text { ennemi de l'homme. }\end{array}$ \\
\hline $\begin{array}{l}\text { Van Vogt Alfred E., Le monde des Ã, (The world of } \tilde{A}, 1945 \text {, version } \\
\text { définitive 1970), J'ai lu, Flammarion, 1973, } 309 \text { p. }\end{array}$ & Télécommunication & $\begin{array}{l}\text { Ouvrage écrit en référence à la } \\
\text { cybernétique de Wiener. Le réseau } \\
\text { centralisé asservi l'homme. Comment } \\
\text { organiser la complexité? }\end{array}$ \\
\hline $\begin{array}{l}\text { Clarke Arthur C., Je me souviens de Babylone, rédigé en } 1960 \text { sur la base } \\
\text { d'un article de 1945. Cité par Georges W. Barlow, "L'odyssée d'Arthur C. } \\
\text { Clarke ", in Arthur C. Clarke, Le livre d'or de la science-fiction, Presses } \\
\text { pocket, 1981, p. } 12 \text {. }\end{array}$ & Télécommunication & $\begin{array}{l}\text { Première proposition de télécommunication } \\
\text { par satellite. Le réseau outil de propagande. }\end{array}$ \\
\hline $\begin{array}{l}\text { Leinster Murray, « Un logique nommé Joe » (A logic named Joe, 1946), in } \\
\text { Histoires de machines, La grande Anthologie de la science-fiction, Le livre } \\
\text { de poche, 1974, pp. 305-326. }\end{array}$ & $\begin{array}{l}\text { Informatique, multimédia, } \\
\text { banque }\end{array}$ & $\begin{array}{l}\text { Le tout est supérieur à la somme des parties } \\
\text { (l'ordinateur en réseau devient intelligent et } \\
\text { échappe à son créateur). }\end{array}$ \\
\hline Orwell Georges, 1984, Londres, 1949, 241 p. & Télécommunication & Le réseau centralisé asservi l'homme. \\
\hline $\begin{array}{l}\text { Sturgeon Theodore, "Les étoiles sont vraiment le Styx » (The stars are the } \\
\text { Styx, 1950), in Amour, impair et manque, 1981, pp. 251-306. }\end{array}$ & Mise en réseau d'êtres humains & $\begin{array}{l}\text { L'amour est un opérateur de réseau. Forte } \\
\text { ubiquité, coordination décentralisée. La } \\
\text { conquête des étoiles. }\end{array}$ \\
\hline $\begin{array}{l}\text { Sturgeon Theodore, Les plus qu'humains (édition originale en anglais, } \\
\text { 1953), J'ai lu Flammarion, 1972, } 307 \text { p. }\end{array}$ & $\begin{array}{l}\text { Mise en réseau d'êtres humains } \\
\text { (télépathie) }\end{array}$ & $\begin{array}{l}\text { Le tout est supérieur à la somme des parties } \\
\text { (les hommes en réseau deviennent des } \\
\text { surhommes). }\end{array}$ \\
\hline $\begin{array}{l}\text { Zélazny Roger, « Les autos sauvages » (Devil car, 1956), in Histoires de } \\
\text { machines, La grande Anthologie de la science-fiction, Le livre de poche, } \\
\text { 1974, pp. 219-239. }\end{array}$ & Automobile & $\begin{array}{l}\text { La révolte de la technique qui échappe à } \\
\text { son créateur. }\end{array}$ \\
\hline $\begin{array}{l}\text { Silverberg Robert, " Les colporteurs de souffrance " (The pain peddlers, } \\
\text { 1963), in Histoires de sociétés futures, La grande Anthologie de la science- } \\
\text { fiction, Le livre de poche, 1984, pp. 221-235. }\end{array}$ & Télécommunication (télévision) & $\begin{array}{l}\text { Les réseaux outils d'asservissement. Le } \\
\text { danger de la télévision réalité. }\end{array}$ \\
\hline $\begin{array}{l}\text { Brown Fredric, «La réponse » (The Answer, 1963), in Histoires de } \\
\text { machines, La grande Anthologie de la science-fiction, Le livre de poche, } \\
\text { 1974, pp. 365-366. }\end{array}$ & Informatique & $\begin{array}{l}\text { Le tout est supérieur à la somme des parties } \\
\text { (I'ordinateur en réseau devient intelligent et } \\
\text { échappe à son créateur). }\end{array}$ \\
\hline $\begin{array}{l}\text { Vance Jack, «L'arche d'Alfred » (Alfred's Ark (1965), in Docteur Bizarre, } \\
\text { Presses pocket, 1992, pp. } 205 \text { et } 228 .\end{array}$ & Transport interplanétaire & $\begin{array}{l}\text { Les réseaux de transport générateurs de } \\
\text { développement territorial. }\end{array}$ \\
\hline $\begin{array}{l}\text { Leiber Fritz, "SOS Médecin " (Anserwing service, 1967), in Histoires } \\
\text { Mécaniques, La grande Anthologie de la science-fiction, Le livre de } \\
\text { poche, 1985, pp.13-23. }\end{array}$ & Télécommunication (téléphone) & $\begin{array}{l}\text { Les réseaux de télécommunication rendent } \\
\text { possibles des actions à distance. }\end{array}$ \\
\hline $\begin{array}{l}\text { Dick Philip K., Blade Runner, J'ai Lu Flammarion, (ed. originale « Do } \\
\text { androids dream of electric sheep? », 1968). }\end{array}$ & $\begin{array}{l}\text { Télécommunication par contact } \\
\text { physique }\end{array}$ & $\begin{array}{l}\text { La prière est un opérateur de réseau. Plus } \\
\text { généralement, dans les romans de Dick, le } \\
\text { thème des mondes virtuels préfigure l'idée } \\
\text { du territoire dans le réseau développé par le } \\
\text { mouvement cyberpunk. }\end{array}$ \\
\hline $\begin{array}{l}\text { Reynolds Mack, «Criminel en utopie» (Criminal in utopia), 1968, in } \\
\text { Histoires fausses, La grande Anthologie de la science-fiction, Le livre de } \\
\text { poche, 1984, pp. 267-296. }\end{array}$ & Cartes banquaires & $\begin{array}{l}\text { Le réseau outil d'asservissement vs le } \\
\text { piratage qui restitue les libertés } \\
\text { individuelles. }\end{array}$ \\
\hline $\begin{array}{l}\text { Houston James D., « Masque à gaz » (Gas masq, 1969), in Histoires de } \\
\text { I'an 2000, La Grande Anthologie de la science-fiction, Le livre de poche, } \\
\text { 1985, pp. 65-79. }\end{array}$ & Automobile & $\begin{array}{l}\text { La saturation des flux et les risques } \\
\text { d'engorgement des réseaux. }\end{array}$ \\
\hline $\begin{array}{l}\text { Goulart Ron, "Le vide-ordure » (Disposal, 1969), in Chambon Jacques, } \\
\text { Dans la cité future, Casterman, 1979, pp. 165-175. }\end{array}$ & $\begin{array}{l}\text { Ramassage des ordures } \\
\text { ménagères }\end{array}$ & $\begin{array}{l}\text { La saturation des flux et les effets pervers de } \\
\text { la régulation des réseaux et de l'écologie }\end{array}$ \\
\hline
\end{tabular}




\begin{tabular}{|c|c|c|}
\hline $\begin{array}{l}\text { Harrison Harry, "Compagnons de chambre » (Roomates, 1970), in } \\
\text { Histoires de l'an 2000, La grande Anthologie de la science-fiction, Le livre } \\
\text { de poche, 1985, pp. 110-141. }\end{array}$ & Distribution de l'eau & $\begin{array}{l}\text { Réseaux des pauvres, réseaux des } \\
\text { riches. La fragilité des gated } \\
\text { communities vis-à-vis des risques de } \\
\text { coupure des réseaux. }\end{array}$ \\
\hline $\begin{array}{l}\text { Lafferty R. A., "La fée interurbaine » (interurban queen, 1970), in Histoires } \\
\text { de la 4e dimension, La grande Anthologie de la science-fiction, Le livre de } \\
\text { poche, 1974, pp. 335-349. }\end{array}$ & Électricité & Les effets pervers de l'écologie. \\
\hline $\begin{array}{l}\text { Maclean Katherine, "L'homme qui avait disparu » (The missing man, } \\
\text { 1971), in Histoires de I'an 2000, La grande Anthologie de la science- } \\
\text { fiction, Le livre de poche, 1985, pp. 178-247. }\end{array}$ & Informatique & $\begin{array}{l}\text { Les réseaux interconnectés sont des } \\
\text { instruments de propagation des } \\
\text { risques. }\end{array}$ \\
\hline $\begin{array}{l}\text { Peck Richard E. «Train de banlieue » (Gantlet, 1972), in Chambon } \\
\text { Jacques, Dans la cité future, Casterman, 1979, pp. 150-164. }\end{array}$ & Transport (train) & $\begin{array}{l}\text { Réseaux des pauvres, réseaux des } \\
\text { riches. La fragilité des gated } \\
\text { communities vis-à-vis des risques de } \\
\text { sabotage des réseaux. }\end{array}$ \\
\hline $\begin{array}{l}\text { Sheckley Robert, "Le prix du danger » (The prize of peril, 1972), in } \\
\text { Histoires de demain, La grande Anthologie de la science-fiction, Le livre } \\
\text { de poche, 1974, pp. 323-349. }\end{array}$ & Télécommunication (télévision & $\begin{array}{l}\text { Les réseaux outils d'asservissement. } \\
\text { Le danger de la télévision réalité. }\end{array}$ \\
\hline $\begin{array}{l}\text { Arthur C. Clarke, Rendez-vous avec Rama, J'ai Lu SF, 1980, } 254 \text { p. } \\
\text { (Rendez-vous with Rama, 1973). }\end{array}$ & Radio & $\begin{array}{l}\text { Les failles de la technique rendent à } \\
\text { I'homme sa liberté de décision. }\end{array}$ \\
\hline $\begin{array}{l}\text { McCaffrey Anne, Le vol de Pégase, (To ride Pegasus, 1973), Presses } \\
\text { Pocket, 1992, } 4 \text { vol. }\end{array}$ & $\begin{array}{l}\text { Communication par télépathie. } \\
\text { Fret. }\end{array}$ & $\begin{array}{l}\text { Le tout est supérieur à la somme des } \\
\text { parties (les hommes en réseau } \\
\text { deviennent des surhommes). La } \\
\text { conquête des étoiles }\end{array}$ \\
\hline $\begin{array}{l}\text { Vance Jack, " La grande Bamboche » (Rumfuddle, 1973), in Bateaux ivres } \\
\text { au fil du temps, Casterman, 1978, p. } 185 .\end{array}$ & $\begin{array}{l}\text { Transport. (déplacement instantané } \\
\text { par translateur de matière) }\end{array}$ & $\begin{array}{l}\text { L'interconnexion au service du } \\
\text { déplacement. }\end{array}$ \\
\hline $\begin{array}{l}\text { Brunner John, Sur l'onde de choc (The schockwave rider, 1975), Le livre } \\
\text { de poche, } 1977,347 \text { p. }\end{array}$ & Informatique & $\begin{array}{l}\text { Le réseau outil d'asservissement vs le } \\
\text { piratage qui restitue les libertés } \\
\text { individuelles. }\end{array}$ \\
\hline $\begin{array}{l}\text { Herbert Franck, "Le syndrome de la Marie-Céleste » (Marie-Celeste move, } \\
\text { 1976) in Histoires de I'an 2000, La grande Anthologie de la science- } \\
\text { fiction, Le livre de poche, 1985, pp. 55-64. }\end{array}$ & Automobile & $\begin{array}{l}\text { Réseaux de transport et aménagement } \\
\text { du territoire. Démotorisation et } \\
\text { écologie. }\end{array}$ \\
\hline $\begin{array}{l}\text { Clarke Arthur C., Les fontaines du paradis, (The Foutains of Paradise, 1979) } \\
\text { Albin Michel, } 1980\end{array}$ & Transport interplanétaire & $\begin{array}{l}\text { La machine circulatoire. La } \\
\text { communication universelle via la } \\
\text { conquête des étoiles. }\end{array}$ \\
\hline $\begin{array}{l}\text { Delaney Joseph, Stiegler Marc, "Valentina », 1983, in Duvic Patrice, } \\
\text { Demain les puces, Denoël, 1996, pp. 61-153. }\end{array}$ & Informatique & $\begin{array}{l}\text { Le réseau outil d'asservissement vs le } \\
\text { piratage qui restitue les libertés } \\
\text { individuelles. Le territoire à l'intérieur } \\
\text { du réseau. }\end{array}$ \\
\hline $\begin{array}{l}\text { Shiner Lewis, "La cité blanche » (White city, 1990), in I. Asimov présente: } \\
\text { Futurs sens dessus dessous, Presse Pocket, 1993, pp. 67-73. }\end{array}$ & Électricité & $\begin{array}{l}\text { Le risque humain dans la gestion des } \\
\text { réseaux. }\end{array}$ \\
\hline $\begin{array}{l}\text { Butler Octavia E., La parabole du semeur, J'ai lu Science-Fiction, 1995, } \\
382 \text { p. (« Parable of the sower», 1993). }\end{array}$ & Télévision, radio, route & $\begin{array}{l}\text { Fragilité des gated communities } \\
\text { quand les réseaux de } \\
\text { télécommunications sont coupés et } \\
\text { qu'elle est assiégée par la route. }\end{array}$ \\
\hline $\begin{array}{l}\text { Dantec Maurice G., Les racines du mal, Gallimard, Série noire, 1995, } \\
636 \text { p. }\end{array}$ & $\begin{array}{l}\text { Transport, télécommunication, } \\
\text { informatique, neurones, etc. }\end{array}$ & $\begin{array}{l}\text { Notre monde est saturé de réseaux, } \\
\text { icones de la modernité. }\end{array}$ \\
\hline Simmons Dan, Hypérion, Presses Pocket, 2000 -ed. originale 1997-6 vol. & $\begin{array}{l}\text { Transport (déplacement instantané par } \\
\text { translateur de matière). } \\
\text { Communication par télépathie }\end{array}$ & $\begin{array}{l}\text { Le réseau outil d'asservissement vs le } \\
\text { piratage qui restitue les libertés } \\
\text { individuelles et la communication } \\
\text { universelle. L'ubiquité est un idéal } \\
\text { que l'on peut atteindre en renonçant } \\
\text { à la technique. }\end{array}$ \\
\hline $\begin{array}{l}\text { Pullman Philip, À la croisée des mondes, Gallimard Jeunesse, } 2001 \text { — (ed. } \\
\text { originale 2000) —, } 3 \text { vol. }\end{array}$ & $\begin{array}{l}\text { Transport (déplacement instantané par } \\
\text { translateur de matière) }\end{array}$ & $\begin{array}{l}\text { L'ubiquité est un idéal impossible à } \\
\text { atteindre. }\end{array}$ \\
\hline $\begin{array}{l}\text { Autres thèmes abordés mais non développés : mouvement cyberpunk, } \\
\text { cyborgs. }\end{array}$ & $\begin{array}{l}\text { Informatique, télécommunication, } \\
\text { connection directe de cerveau à } \\
\text { ordinateur, etc. }\end{array}$ & $\begin{array}{l}\text { Cyberpunk : Le territoire est à } \\
\text { l'intérieur du réseau. La connexion } \\
\text { est généralisée. L'ubiquité est } \\
\text { possible. Le hacker est très présent. } \\
\text { Cyborgs : la connection } \\
\text { homme/machine est directe. }\end{array}$ \\
\hline
\end{tabular}

